\title{
Quantum Group Invariants and Link Polynomials
}

\author{
R. B. Zhang, M. D. Gould and A. J. Bracken \\ Department of Mathematics, The University of Queensland, Brisbane, Qld. 4072, Australia
}

Received December 3, 1989; in revised form August 1, 1990

\begin{abstract}
A general method is developed for constructing quantum group invariants and determining their eigenvalues. Applied to the universal $R$-matrix this method leads to the construction of a closed formula for link polynomials. To illustrate the application of this formula, the quantum groups $U_{q}\left(E_{8}\right), U_{q}(s o(2 m+1))$ and $U_{q}(g l(m))$ are considered as examples, and corresponding link polynomials are obtained.
\end{abstract}

\section{Introduction}

Quantum groups $[1,2]$ play a fundamental role in the theory of integrable models [3], conformal field theory [4], and the classification of knots and links [5-10]. It appears possible that they may provide the key to understanding the intimate relationship between integrable systems and knot theory $[8,9]$, and even the more basic problem of why integrable models exist [8].

It is well known that to a large extent the study of a physical theory involves the exploration of its symmetries. In particular, it is desirable to determine the invariants of the symmetry algebra of the theory, which usually correspond to certain physical observables. Quantum groups arise as underlying symmetries of integrable lattice models and conformal field theory, and their invariants therefore are of crucial importance to the understanding of these problems. The quantum group invariants, being quantum analogs of the Gelfand invariants of ordinary Lie algebras, are also extremely useful for characterizing quantum group representations.

In this paper, we will develop a general method for constructing quantum group invariants and determining their eigenvalues. As a natural application we apply the method to Drinfeld's [2] universal $R$-matrix to obtain certain quantum group invariants, which in turn enable us to construct a closed formula for link polynomials. Our formula agrees with that of Reshetikhin [10] obtained through a completely different approach, but is more explicit, and our derivation is also more straightforward. To illustrate how the general formula works, we study as 
examples the quantum groups $U_{q}(g l(m)), U_{q}(\operatorname{so}(2 m+1))$ and $U_{q}\left(E_{8}\right)$ in certain representations.

The structure of the paper is as follows. In Sect. 2 we develop a general theory for constructing quantum group invariants, and in Sect. 3 we use the theory to construct a general and closed formula for link polynomials. In Sect. 4 we present concrete examples of link polynomials and in the final section we briefly discuss the main results of the paper.

\section{Quantum Group Invariants}

Corresponding to each simple Lie algebra $g$, there exists a quantum group $U_{q}(g)$ defined as follows in terms of generators $e_{i}, f_{i} \in U_{q}(g), i=1,2, \ldots, r$ associated with the simple roots $\alpha_{i}, i=1,2, \ldots, r$ of $g$, and corresponding Cartan generators $h_{i}$, $i=1,2, \ldots, r$. Let $(\cdot, \cdot)$ be the invariant bilinear form for the weight space $H^{*}$, the dual of the vector space $H$ spanned by the $h_{i}$ 's, and let $A=\left(a_{i j}\right)$ be the Cartan matrix of $g$. For a nonzero parameter $q \in C$ we define $q_{i}=q^{\left(\alpha_{i}, \alpha_{i}\right) / 2}, K_{i}=q^{h_{i}}, i=1,2, \ldots, r$; then $U_{q}(g)$ is generated by $\left\{K_{i}^{ \pm 1}, e_{i}, f_{i} \mid i=1,2, \ldots, r\right\}$, with

$$
\begin{aligned}
& {\left[h_{i}, h_{j}\right]=0,} \\
& K_{i} e_{j} K_{i}^{-1}=q_{i}^{a_{i j}} e_{j}, \quad K_{i} f_{j} K_{i}^{-1}=q_{i}^{-a_{i j}} f_{j}, \\
& {\left[e_{i}, f_{j}\right]=\delta_{i j}\left(K_{i}-K_{i}^{-1}\right) /\left(q_{i}-q_{i}^{-1}\right),} \\
& \sum_{t=0}^{1-a_{i j}}(-)^{t}\left[\begin{array}{c}
1-a_{i j} \\
t
\end{array}\right]_{q_{i}} e_{i}^{1-a_{i j}-t} e_{j} e_{i}^{t}=0, \quad i \neq j, \\
& \sum_{t=0}^{1-a_{i j}}(-)^{t}\left[\begin{array}{c}
1-\alpha_{i j} \\
t
\end{array}\right]_{q_{i}} f_{i}^{1-a_{i j}-t} f_{j} f_{i}^{t}=0, \quad i \neq j,
\end{aligned}
$$

where

$$
\left[\begin{array}{c}
m \\
n
\end{array}\right]_{q}=\left\{\begin{array}{lr}
\frac{\left(q^{m}-q^{-m}\right)\left(q^{m-1}-q^{1-m}\right) \cdots\left(q^{m-n+1}-q^{n-m-1}\right)}{\left(q-q^{-1}\right)\left(q^{2}-q^{-2}\right) \cdots\left(q^{n}-q^{-n}\right)}, & m>n>0 \\
1, & n=m, 0 \\
0, & \text { otherwise. }
\end{array}\right.
$$

A coproduct structure $\Delta: U_{q}(g) \rightarrow U_{q}(g) \otimes U_{q}(g)$ exists, which is defined by

$$
\begin{aligned}
& \Delta\left(h_{i}\right)=h_{i} \otimes 1+1 \otimes h_{i}, \\
& \Delta\left(e_{i}\right)=e_{i} \otimes q^{-h_{i} / 2}+q^{h_{i} / 2} \otimes e_{i}, \\
& \Delta\left(f_{i}\right)=f_{i} \otimes q^{-h_{i} / 2}+q^{h_{i} / 2} \otimes f_{i},
\end{aligned}
$$

and it can easily be checked that $\Delta$ does indeed define an algebra homomorphism. Replacing $q$ by $q^{-1}$ in (2), we obtain another algebra homomorphism $\bar{\Delta}$. Both $\Delta$ and $\bar{\Delta}$ can be extended uniquely to the entire Hopf algebra $U_{q}(g)$.

Consider a finite dimensional irreducible representation of $U_{q}(g)$ furnished by the irreducible module $V\left(\Lambda_{0}\right)$ with $\Lambda_{0}$ being the highest weight. Given an operator 
$\Gamma \in U_{q}(g) \otimes \operatorname{End}\left(V\left(\Lambda_{0}\right)\right)$ satisfying

$$
\begin{aligned}
{\left[\Gamma, \Delta\left(K_{i}^{ \pm 1}\right)\right] } & =0, \\
{\left[\Gamma, \Delta\left(e_{i}\right)\right] } & =0, \\
{\left[\Gamma, \Delta\left(f_{i}\right)\right] } & =0, \quad \forall i,
\end{aligned}
$$

we want to construct from $\Gamma$ an operator $C \in U_{q}(g)$, such that

$$
\begin{aligned}
{\left[C, K_{i}^{ \pm 1}\right] } & =0, \\
{\left[C, e_{i}\right] } & =0, \\
{\left[C, f_{i}\right] } & =0, \quad \forall i .
\end{aligned}
$$

Let $\rho$ denote the half-sum of positive roots of $g$, and let $\tau_{i}$ be the operation of taking the partial trace of any operator belonging to $\operatorname{End}\left(V_{1} \otimes V_{2} \otimes \cdots \otimes V_{l}\right)$, over the $i$-th space in the tensor product. Denoting by $h_{\rho} \in U_{q}(g)$ the Cartan element satisfying $\alpha\left(h_{\rho}\right)=(\alpha, \rho) \forall \alpha \in H^{*}$, we have the following

Proposition 1. Given a $\Gamma$ satisfying (3), the operator $C \in U_{q}(g)$ given by

$$
C=\tau_{2}\left\{\left(1 \otimes q^{-2 h_{\rho}}\right) \Gamma\right\}
$$

satisfies Eqs. (4).

Proof. Consider the first equation in (3). Multiplying it by $1 \otimes q^{-\left(2 h_{\rho} \pm h_{i}\right)}$ then taking the partial trace $\tau_{2}$, we obtain

$$
\begin{aligned}
0 & =\tau_{2}\left\{\left[\Gamma, \Delta\left(K_{i}^{ \pm 1}\right)\right]\left(1 \otimes q^{-\left(2 h_{\rho} \pm h_{i}\right)}\right)\right\} \\
& =\left[\tau_{2}\left\{\left(1 \otimes q^{-2 h_{\rho}}\right) \Gamma\right\}, K_{i}^{ \pm 1}\right],
\end{aligned}
$$

which is (4a). The first and second equations of (3) together lead to

$$
0=\tau_{2}\left\{\left[\Gamma, \Delta\left(e_{i}\right) \Delta\left(K_{i}^{-1 / 2}\right)\right]\left(1 \otimes q^{-\left(2 h_{\rho}-h_{i}\right)}\right)\right\},
$$

which yields

$$
\begin{aligned}
& {\left[\tau_{2}\left\{\left(1 \otimes q^{-2 h_{\rho}}\right) \Gamma\right\}, e_{i} K_{i}^{-1 / 2}\right]} \\
& \quad+\tau_{2}\left\{\Gamma\left(1 \otimes e_{i} q^{-\left(2 h_{\rho}-h_{i} / 2\right)}\right)\right\} \\
& \quad-\tau_{2}\left\{\left(1 \otimes e_{i} q^{-h_{i} / 2}\right) \Gamma\left(1 \otimes q^{-\left(2 h_{\rho}-h_{i}\right)}\right)\right\}=0 .
\end{aligned}
$$

The last term of (6) can be rewritten as

$$
\tau_{2}\left\{\left(1 \otimes q^{-\left(2 h_{\rho}-h_{i}\right)} e_{i} q^{-h_{i} / 2}\right) \Gamma\right\},
$$

and by using the fact that

$$
\left[e_{i}, q^{-\left(2 h_{\rho}-h_{i}\right)}\right]=0
$$

we can simplify it further to

$$
\tau_{2}\left\{\left(1 \otimes e_{i} q^{-\left(2 h_{\rho}-h_{i} / 2\right)}\right) \Gamma\right\} .
$$

Therefore, the second and third terms in (6) cancel each other, and we obtain

$$
\left[\tau_{2}\left\{\left(1 \otimes q^{-2 h_{\rho}}\right) \Gamma\right\}, e_{i}\right]=0 .
$$


In exactly the same way we can show that $(4 c)$ holds. Thus we have proved the proposition.

Equations $(4 \mathrm{a}-\mathrm{c})$ guarantee that $C \in U_{q}(g)$ takes a constant value on any irreducible $U_{q}(g)$ module, thus can be called an invariant of the quantum group $U_{q}(g)$. As a corollary of the proposition, we have

Corollary 1. Given

$$
\Gamma \in \overbrace{U_{q}(g) \otimes \cdots \otimes U_{q}}^{k-1}) \otimes \operatorname{End}\left(V\left(\Lambda_{0}\right)\right)
$$

such that

$$
\left[\Gamma, \Delta^{(k)}(u)\right]=0 \quad \forall u \in U_{q}(g)
$$

then

$$
C \in \overbrace{U_{q}(g) \otimes \cdots \otimes U_{q}}^{k-1}(g)
$$

defined by

$$
C=\Delta^{(k-1)}\left(q^{2 h_{\rho}}\right) \tau_{n}\left\{\Delta^{(k)}\left(q^{-2 h_{\rho}}\right) \Gamma\right\}
$$

satisfies

$$
\left[C, \Delta^{(k-1)}(u)\right]=0 \quad \forall u \in U_{q}(g),
$$

where we have adopted the notation

$$
\Delta^{(i)}=(\Delta \otimes \overbrace{I \otimes \cdots \otimes I}^{i-2})(\Delta \otimes I \otimes \overbrace{\overbrace{\otimes \otimes}}^{i-3} I) \cdots(\Delta \otimes I) \Delta, \quad i \geqq 2 .
$$

To evaluate the eigenvalue of any $C$ on an irreducible $U_{q}(g)$ module $V(\Lambda)$, we multiply $C$ by $q^{-2 h_{\rho}}$, then take the trace over $V(\Lambda)$,

$$
\operatorname{tr}_{V(\Lambda)}\left(q^{-2 h_{\rho}} C\right)=c(\Lambda) \operatorname{tr}_{V(\Lambda)}\left(q^{-2 h_{\rho}}\right)
$$

where $c(\Lambda)$ is the eigenvalue of $C$ on $V(\Lambda)$. On the other hand,

$$
\operatorname{tr}_{V(\Lambda)}\left(q^{-2 h_{\rho}} C\right)=\operatorname{tr}_{V(\Lambda) \otimes V\left(\Lambda_{0}\right)}\left\{\Delta\left(q^{-2 h_{\rho}}\right) \Gamma\right\} .
$$

Introducing the $q$-dimension $D_{q}(\Lambda)$ of the irreducible $U_{q}(g)$ module $V(\Lambda)$ defined by

$$
D_{q}(\Lambda)=\operatorname{tr}_{V(\Lambda)}\left(q^{-2 h_{\rho}}\right)
$$

we obtain from (9) and (10)

Proposition 2. Given a $\Gamma$ as defined by (3), the operator $C$ defined by (5) takes the eigenvalue

$$
c(\Lambda)=\operatorname{tr}_{V(\Lambda) \otimes V\left(\Lambda_{0}\right)}\left\{\Delta\left(q^{-2 h_{\rho}}\right) \Gamma\right\} / D_{q}(\Lambda)
$$

on the irreducible $U_{q}(g)$ module $V(\Lambda)$.

The $q$-dimension of an irreducible $U_{q}(g)$ module $V(\Lambda)$ can actually be evaluated explicitly. Note that $D_{q}(\Lambda)$ can be written as

$$
D_{q}(\Lambda)=\sum_{\lambda} m_{\lambda} q^{-2(\rho, \lambda)}
$$


where $m_{\lambda}$ is the multiplicity of the weight $\lambda$, and the sum is over the entire weight spectrum of $V(\Lambda)$. It is known that $V(\Lambda)$ has the same weight spectrum as the $g$ module with the same highest weight $\Lambda[10,11]$ so we can rewrite (13) as

$$
D_{q}(\Lambda)=\prod_{\alpha \in \Phi^{+}}\left[q^{-(\rho, \alpha)}-q^{(\rho, \alpha)}\right]^{-1} \sum_{\sigma \in W} \operatorname{det} \sigma q^{-(\sigma(\Lambda+\rho), 2 \rho)},
$$

where $\Phi^{+}$and $W$ are respectively the set of positive roots and the Weyl group of the Lie algebra $g$. Using the Weyl group invariance of the bilinear form $(\cdot, \cdot)$ and the fact that $\operatorname{det} \sigma=\operatorname{det}\left(\sigma^{-1}\right) \forall \sigma \in W$, we can turn the sum on the right-hand side of (14) into

$$
\sum_{\sigma \in W} \operatorname{det} \sigma q^{-(\sigma(\Lambda+\rho), 2 \rho)}=\sum_{\sigma \in W} \operatorname{det} \sigma q^{-2(\sigma(\rho), \Lambda+\rho)} .
$$

Upon using Weyl's denominator formula we obtain

$$
\sum_{\sigma \in W} \operatorname{det} \sigma q^{-(\sigma(\Lambda+\rho), 2 \rho)}=\prod_{\alpha \in \Phi^{+}}\left[q^{-(\Lambda+\rho, \alpha)}-q^{(\Lambda+\rho, \alpha)}\right] .
$$

Applying (15) to Eq. (14) we arrive at

Lemma 1. The q-dimension of the irreducible $U_{q}(g)$ module $V(\Lambda)$ is given by

$$
D_{q}(\Lambda)=\prod_{\alpha \in \Phi^{+}} \frac{q^{(\Lambda+\rho, \alpha)}-q^{-(\Lambda+\rho, \alpha)}}{q^{(\rho, \alpha)}-q^{-(\rho, \alpha)}},
$$

where $\Phi^{+}$denotes the set of positive roots of the simple Lie algebra $g$.

Note that in the limit $q \rightarrow 1,(16)$ reduces to the ordinary Weyl dimension formula.

Now we consider an example. Assume that the $U_{q}(g)$ in the product $U_{q}(g) \otimes$ $\operatorname{End}\left(V\left(\Lambda_{0}\right)\right)$ acts in an irreducible representation furnished by the module $V(\Lambda)$. Define $P_{\mu}$ to be the operator which projects the tensor product $V(\Lambda) \otimes V\left(\Lambda_{0}\right)$ into the irreducible $U_{q}(g)$ module $V\left(\Lambda_{\mu}\right) \subset V(\Lambda) \otimes V\left(\Lambda_{0}\right)$, i.e.

$$
P_{\mu}\left(V(\Lambda) \otimes V\left(\Lambda_{0}\right)\right)=V\left(\Lambda_{\mu}\right), \quad P_{\mu}^{2}=P_{\mu} .
$$

Then it is obvious that $P_{\mu}$ satisfies Eqs. (3). Let

$$
C_{\mu}=\tau_{2}\left\{\left(1 \otimes q^{-2 h_{\rho}}\right) P_{\mu}\right\}
$$

and denote its eigenvalue on $V(\Lambda)$ by $c_{\mu}(\Lambda)$. Then following Proposition 2 , we have

$$
D_{q}(\Lambda) c_{\mu}(\Lambda)=\operatorname{tr}_{V(\Lambda) \otimes V\left(\Lambda_{0}\right)}\left[\Delta\left(q^{\left.-2 h_{\rho}\right)} P_{\mu}\right]\right.
$$

Note that the right-hand side of Eq. (19) is nothing but the q-dimension of $V\left(\Lambda_{\mu}\right)$; thus we have

Lemma 2. The operator $C_{\mu}$ defined by (18) takes on $V(\Lambda)$ the eigenvalue

$$
\begin{aligned}
c_{\mu}(\Lambda) & =D_{q}\left(\Lambda_{\mu}\right) / D_{q}(\Lambda) \\
& =\prod_{\alpha \in \Phi^{+}} \frac{q^{\left(\Lambda_{\mu}+\rho, \alpha\right)}-q^{-\left(\Lambda_{\mu}+\rho, \alpha\right)}}{q^{(\Lambda+\sigma, \alpha)}-q^{-(\Lambda+\rho, \alpha)}} .
\end{aligned}
$$

\section{Link Polynomials}

In this section we will apply the results of the last section to Drinfeld's universal $R$-matrix, in order to construct link polynomials. In particular we will define a 
Markov trace on the braid group representation generated by the universal $R$-matrix, and use this Markov trace to develop a closed formula for link polynomials.

Let us first briefly describe the universal $R$-matrix obtained by Drinfeld [2]. Consider the Hopf subalgebras $U_{q}\left(b_{+}\right), U_{q}\left(b_{-}\right) \subset U_{q}(g)$ generated by $\left\{h_{i}, e_{i} \mid i=\right.$ $1,2, \ldots, r\}$ and $\left\{h_{i}, f_{i} \mid i=1,2, \ldots, r\right\}$ respectively. Define a basis $\left\{e_{s} \mid s=1,2, \ldots\right\}$ for $U_{q}\left(b_{-}\right)$; then there exists a basis $\left\{e^{s} \mid s=1,2, \ldots\right\}$ dual to $\left\{e_{s} \mid s=1,2, \ldots\right\}$ for the Hopf subalgebra $U_{q}\left(b_{+}\right)$. The operator $R$ defined by

$$
R=\sum_{s} e_{s} \otimes e^{s} \in U_{q}(g) \otimes U_{q}(g)
$$

satisfies the following equations

$$
R \Delta(u)=\bar{\Delta}(u) R, \quad \forall u \in U_{q}(g)
$$

and

$$
R_{12} R_{13} R_{23}=R_{23} R_{13} R_{12},
$$

where $\Delta$ and $\bar{\Delta}$ are defined in Sect. 2, and $R_{12}$ etc. are given by

$$
R_{12}=\sum_{s} e_{s} \otimes e^{s} \otimes I, \quad R_{13}=\sum_{s} e_{s} \otimes I \otimes e^{s}, \quad R_{23}=\sum_{s} I \otimes e_{s} \otimes e^{s} .
$$

For the purpose of building braid group representations, we are only interested in the special case when both $U_{q}(g)$ 's in (21) act in the same irreducible representation furnished by a module $V(\Lambda)$, i.e. $R \in \operatorname{End}(V(\Lambda) \otimes V(\Lambda))$. In this case, we can define a permutation operator $P$ such that

$$
P(|\mu\rangle \otimes|v\rangle)=|v\rangle \otimes|\mu\rangle \quad \forall|\mu\rangle \otimes|v\rangle \in V(\Lambda) \otimes V(\Lambda) .
$$

Let

$$
\sigma=P R .
$$

Then Eqs. (22) and (23) can be written in terms of $\sigma$ as

$$
[\sigma, \Delta(u)]=0, \quad \forall u \in U_{q}(g),
$$

and

$$
(I \otimes \sigma)(\sigma \otimes I)(I \otimes \sigma)=(\sigma \otimes I)(I \otimes \sigma)(\sigma \otimes I) .
$$
It follows immediately that the operators $\sigma_{i} \in \operatorname{End}\left(\bigotimes_{1}^{n} V(\Lambda)\right), \quad i \in\{1,2, \ldots, n-1\}$
defined by

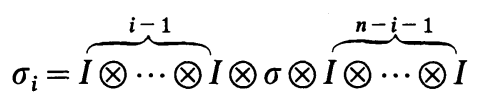

generate a nontrivial representation of the rank $(n-1)$ braid group $\mathfrak{B}_{n}$. In the remainder of the paper, we will use $B_{n}$ to denote this representation.

Let $h$ be an arbitrary Cartan element of $U_{q}(g)$ and define

$$
\Delta^{(k)}\left(q^{h}\right)=q^{h} \otimes q^{h} \otimes \cdots \otimes q^{h} \in \operatorname{End}\left(\bigotimes_{1}^{k} V(\Lambda)\right) .
$$

Then we have the following 
Proposition 3. Define

$$
\phi(\theta)=\operatorname{tr}\left[\Delta^{(n)}\left(q^{-2 h_{\rho}}\right) \theta\right] / \operatorname{tr}\left[\Delta^{(n)}\left(q^{-2 h_{\rho}}\right)\right],
$$

where $\theta \in B_{n}$ is a word in the generators $\sigma_{i}, i=1,2, \ldots, n-1$, and the trace is taken over the $n$-fold tensor product space. Then $\phi$ has the following Markov properties:

$$
\begin{aligned}
& \text { I: } \quad \phi(\theta \eta)=\phi(\eta \theta), \quad \forall \theta, \eta \in B_{n} ; \\
& \text { II: } \phi\left(\theta \sigma_{n-1}\right)=z \phi(\theta), \quad \forall \theta \in B_{n-1}<B_{n} \text {, } \\
& \phi\left(\theta \sigma_{n-1}^{-1}\right)=\bar{z} \phi(\theta), \quad \forall \theta \in B_{n-1}<B_{n},
\end{aligned}
$$

with $z$ and $\bar{z}$ defined by (33) below.

Proof. In order to prove property I, we note that

$$
\left[\theta, \Delta^{(n)}\left(q^{-2 h_{\rho}}\right)\right]=0, \quad \forall \theta \in B_{n},
$$

which follows directly from (22a). Therefore for any $\theta, \eta \in B_{n}$,

$$
\operatorname{tr}\left[\Delta^{(n)}\left(q^{-2 h_{\rho}}\right) \theta \eta\right]=\operatorname{tr}\left[\Delta^{(n)}\left(q^{-2 h_{\rho}}\right) \eta \theta\right]
$$

which immediately leads to (29). To prove property II, we observe that

$$
\phi\left(\sigma_{i}\right)=z, \quad \phi\left(\sigma_{i}^{-1}\right)=\bar{z}, \quad \forall i \in\{1,2, \ldots, n-1\},
$$

with $z$ and $\bar{z}$ defined respectively by

$$
\begin{aligned}
& z=\operatorname{tr}\left[\Delta\left(q^{-2 h_{\rho}}\right) \sigma\right] / \operatorname{tr}\left[\Delta\left(q^{-2 h_{\rho}}\right)\right], \\
& \bar{z}=\operatorname{tr}\left[\Delta\left(q^{-2 h_{\rho}}\right) \sigma^{-1}\right] / \operatorname{tr}\left[\Delta\left(q^{-2 h_{\rho}}\right)\right] .
\end{aligned}
$$

For an arbitrary $\theta \in B_{n-1}<B_{n}$,

$$
\phi\left(\theta \sigma_{n-1}\right)=\frac{\operatorname{tr}\left\{\tau_{n}\left[\Delta^{(n)}\left(q^{-2 h_{\rho}}\right) \theta \sigma_{n-1}\right]\right\}}{\operatorname{tr}\left\{\tau_{n}\left[\Delta^{(n)}\left(q^{-2 h_{\rho}}\right)\right]\right\}} .
$$

On the right-hand side of the above equation, tr represents the trace taken over the first $(n-1)$ factor spaces in the tensor product $\bigotimes_{1}^{n} V(\Lambda)$ and $\tau_{n}$ is the partial trace taken over the last factor space. By using Proposition 1, we immediately see that

$$
\phi\left(\theta \sigma_{n-1}\right)=z \phi(\theta), \quad \theta \in B_{n-1}<B_{n} .
$$

In exactly the same way we can show that

$$
\phi\left(\theta \sigma_{n-1}^{-1}\right)=\bar{z} \phi(\theta), \quad \theta \in B_{n-1}<B_{n} .
$$

Therefore $\phi$ possesses the Markov properties, and thus qualifies as a Markov trace.

At this point we should point out the connection of our construction as described above, with the work of Turaev [5]. If we let $\mu=q^{-2 h_{\rho}}, \alpha=z D_{q}(\Lambda)$ and $\beta=D_{q}(\Lambda)$, then the collection $(R ; \mu: V(\Lambda) \rightarrow V(\Lambda) ; \alpha, \beta \in \mathbb{C})$ defines an enhanced Yang-Baxter operator in his terminology, and our partial trace $\tau_{i}$ corresponds to his operator trace.

Now we turn to the evaluation of $z$ and $\bar{z}$ defined in (33). Following Proposition 1,

$$
C=\tau_{2}\left\{\left(1 \otimes q^{-2 h_{\rho}}\right) \sigma\right\}
$$


is an invariant, so that

$$
z=c(\Lambda) / D_{q}(\Lambda)
$$

where $c(\Lambda)$ is the eigenvalue of $C$ on $V(\Lambda)$, and $D_{q}(\Lambda)$ is the $q$-dimension of $V(\Lambda)$, which is given explicitly in Sect. 2 .

Let us formally introduce a basis for $V(\Lambda)$ consisting of eigenvectors $|\mu\rangle$ of the Cartan elements, orthogonal under an inner product $\langle\cdot \mid \cdot\rangle$, i.e.

$$
\langle\mu \mid v\rangle=\delta_{\mu v} \text {. }
$$

Denote the lowest weight vector of $V(\Lambda)$ by $|\bar{\Lambda}\rangle$ and consider

$$
\left\langle\bar{\Lambda}\left|q^{-2 h_{\rho}} C\right| \bar{\Lambda}\right\rangle=q^{-2(\rho, \bar{\Lambda})} c(\Lambda) .
$$

The lowest weight $\bar{\Lambda}$ of $V(\Lambda)$ is related to the highest weight $\Lambda$ through the equation

$$
\bar{\Lambda}=\tau(\Lambda), \quad \tau \in W,
$$

where $\tau$ is the unique element of $W$ which turns all the positive roots of $g$ to negative ones. Using (21) and (25) we can also write the left-hand side of (40) as

$$
\begin{aligned}
\sum_{v} & \left\langle\bar{\Lambda} \otimes v\left|\Delta\left(q^{-h_{\rho}}\right) P\left(\sum_{s} e_{s} \otimes e^{s}\right)\right| \bar{\Lambda} \otimes v\right\rangle \\
& =\sum_{v, s}\left\langle v \otimes \bar{\Lambda}\left|q^{-2 h_{\rho}} e_{s} \otimes q^{-2 h_{\rho}} e^{s}\right| \bar{\Lambda} \otimes v\right\rangle \\
& =\sum_{s}\left\langle\bar{\Lambda}\left|q^{-2 h_{\rho}} e^{s} q^{-2 h_{\rho}} e_{s}\right| \bar{\Lambda}\right\rangle .
\end{aligned}
$$

Therefore

$$
q^{-2(\rho, \bar{\Lambda})} c(\Lambda)=\sum_{s}\left\langle\bar{\Lambda}\left|q^{-2 h_{\rho}} e^{s} q^{-2 h_{\rho}} e_{s}\right| \bar{\Lambda}\right\rangle .
$$

Recalling that $\left\{e_{s}\right\}$ is the basis of $U_{q}\left(b_{-}\right)$and $\left\{e^{s}\right\}$ is that for $U_{q}\left(b_{+}\right)$, we immediately see that only those $e_{s}$ and $e^{s}$ made up entirely of Cartan elements of $U_{q}(g)$ contribute to the inner product in (41). Bases for such elements of $U_{q}\left(b_{-}\right)$and $U_{q}\left(b_{+}\right)$are given respectively by

and

$$
\prod_{i=1}^{r}\left(h_{i}\right)^{m_{i}} /\left(m_{i}\right) !, \quad m_{i} \in Z^{+}
$$

$$
\prod_{i=1}^{r}\left(h^{i}\right)^{m_{i}}(\ln q)^{m_{i}}, \quad m_{i} \in Z^{+}
$$

where $h^{i}$ is defined by

$$
\sum_{i=1}^{r} \alpha\left(h^{i}\right) \beta\left(h_{i}\right)=(\alpha, \beta) \quad \forall \alpha, \beta \in H^{*} .
$$

Using this basis in (41) we obtain

$$
q^{-2(\rho, \bar{\Lambda})} c(\Lambda)=q^{-4(\rho, \bar{\Lambda})} \sum_{\left\{m_{i}\right\}}\left\langle\bar{\Lambda}\left|\prod_{i=1}^{r} \frac{\left(h_{i} h^{i} \ln q\right)^{m_{i}}}{\left(m_{i}\right) !}\right| \bar{\Lambda}\right\rangle,
$$

where the sum is over $Z^{+}$for each $m_{i}, i=1,2, \ldots, r$. This sum can easily be seen 
to equal

$$
\left\langle\bar{\Lambda}\left|q^{\sum_{i=1}^{r} h^{i} h_{i}}\right| \bar{\Lambda}\right\rangle
$$

which reduces to $q^{(\bar{\Lambda}, \bar{\Lambda})}$, and using this result in (42) we obtain

i.e.

$$
q^{-2(\rho, \bar{\Lambda})} c(\Lambda)=q^{(\bar{\Lambda}, \bar{\Lambda})-4(\rho, \bar{\Lambda})}
$$

$$
c(\Lambda)=q^{I_{2}(\Lambda)}, \quad I_{2}(\Lambda)=(\Lambda+2 \rho, \Lambda) .
$$

Note that $I_{2}(\Lambda)$ is the value taken by the quadratic Casimir operator of $g$ in the representation with highest weight $\Lambda$.

We point out that in deriving (44), the formal inner product $\langle\cdot \mid \cdot\rangle$ and the basis $\{|\mu\rangle\}$ for $V(\Lambda)$ are introduced only as a computational device; the final result does not depend on the existence of such an inner product or basis.

Using the fact [10] that when $q \rightarrow q^{-1}$, the $R$-matrix is transformed into a matrix similar to $R^{-1}$, we can similarly evaluate $\bar{z}$ to obtain

Lemma 3.

$$
z=q^{I_{2}(\Lambda)} / D_{q}(\Lambda), \quad \bar{z}=q^{-I_{2}(\Lambda)} / D_{q}(\Lambda), \quad I_{2}(\Lambda)=(\Lambda+2 \rho, \Lambda) .
$$

With Proposition 3 and Lemma 3 we are now ready to construct a link polynomial.

Theorem. Let

$$
L(\hat{\theta})=\left[D_{q}(\Lambda)\right]^{n-1} q^{-e(\theta) I_{2}(\Lambda)} \phi(\theta), \quad \theta \in B_{n},
$$

where $\hat{\theta}$ is the link obtained by closing the braid $\theta$, and $e(\theta)$ is the sum of exponents of the $\sigma_{i}$ 's appearing in $\theta \in B_{n}$. Then $L$ defines a link polynomial.

Proof. Equation (46) is equivalent to

$$
L(\hat{\theta})=(z \cdot \bar{z})^{-(n-1) / 2}\left(\frac{\bar{z}}{z}\right)^{e(\theta) / 2} \phi(\theta), \quad \theta \in B_{n} .
$$

It can easily be checked that the $L(\hat{\theta})$ defined by (46a) has the following properties:

$$
\begin{array}{lll}
\mathrm{I}: & L(\widehat{\theta \eta})=L(\widehat{\eta \theta}), & \theta, \eta \in B_{n}, \\
\mathrm{II}: & L\left(\widehat{\theta \sigma_{n-1}}\right)=L(\hat{\theta}), & \theta \in B_{n-1}<B_{n}, \\
& L\left(\widehat{\theta \sigma_{n-1}^{-1}}\right)=L(\hat{\theta}), & \theta \in B_{n-1}<B_{n},
\end{array}
$$

and thus defines a link polynomial.

The formula (46) agrees with that obtained by Reshetikhin [10] using the quantum analogs of Clebsch-Gordan coefficients, but is more explicit. Furthermore, our derivation has avoided lengthy manipulations with such coefficients.

We now examine some of the properties of the link polynomials (46). Consider $\theta=\theta_{1} \theta_{2} \in B_{n}$, where $\theta_{1}$ is composed from generators $\sigma_{1}, \sigma_{2}, \ldots, \sigma_{i-1}$ only, and $\theta_{2}$ from $\sigma_{i}, \sigma_{i+1}, \ldots, \sigma_{n-1}$ only. We express the associated link $\hat{\theta}$ obtained by closing the braid $\theta$ as

$$
\hat{\theta}=\hat{\theta}_{1} \# \hat{\theta}_{2}
$$


where $\hat{\theta}_{1}$ and $\hat{\theta}_{2}$ are regarded as links arising from closing $\theta_{1} \in B_{i}$ and $\theta_{2} \in B_{n-i}$ respectively. When $\theta_{1}$ involves $\sigma_{i-1}$ and $\theta_{2}$ involves $\sigma_{i}$, this coincides with the connected sum of the links $\hat{\theta}_{1}$ and $\hat{\theta}_{2}$ as defined in [12].

It follows readily from the corollary to Proposition 1 that

$$
\phi(\theta)=\phi\left(\theta_{1}\right) \phi\left(\theta_{2}\right)
$$

and then by noting that

$$
e(\theta)=e\left(\theta_{1}\right)+e\left(\theta_{2}\right)
$$

we obtain the following

Lemma 4. Let $\hat{\theta}=\hat{\theta}_{1} \# \hat{\theta}_{2}$, where the links $\hat{\theta}_{1}$ and $\hat{\theta}_{2}$ are the closures of $\theta_{1} \in B_{i}$ and $\theta_{2} \in B_{n-i}$. Then

$$
L(\hat{\theta})=L\left(\hat{\theta}_{1}\right) L\left(\hat{\theta}_{2}\right)
$$

Obviously the lemma can be extended to the case of more than two links.

When

$$
V(\Lambda) \otimes V(\Lambda)=\bigoplus_{\mu=1}^{M} V\left(\Lambda_{\mu}\right)
$$

is multiplicity-free, the braid generator $\sigma \in \operatorname{End}(V(\Lambda) \otimes V(\Lambda))$ assumes a particularly simple form $[10,13]$ :

$$
\sigma=\sum_{\mu=1}^{M} \alpha\left(\Lambda_{\mu}\right) P_{\mu}
$$

with

$$
\alpha\left(\Lambda_{\mu}\right)=\varepsilon\left(\Lambda_{\mu}\right) q^{I_{2}\left(\Lambda_{\mu}\right) / 2-I_{2}(\Lambda)} \quad \mu=1,2, \ldots, M,
$$

where $\varepsilon\left(\Lambda_{\mu}\right)$ is the eigenvalue of the operator $P$ of $(24)$ on $V\left(\Lambda_{\mu}\right)$, in the limit $q \rightarrow 1$. It is easy to see that $\sigma$ satisfies the polynomial identity

$$
\prod_{\mu=1}^{M}\left[\sigma-\alpha\left(\Lambda_{\mu}\right)\right]=\sigma^{M}-\sum_{l=0}^{M-1} A_{l} \sigma^{l}=0,
$$

where the $A_{l}$ are determined by expanding the product on the left-hand side. This leads to the generalized skein relations for (46). For example, with

then

$$
\theta=\theta_{1}\left(\sigma_{i}\right)^{M} \theta_{2}, \quad \theta_{1}, \theta_{2} \in B_{n}
$$

$$
L(\widehat{\theta})=\sum_{l=0}^{M-1} A_{l} L\left(\widehat{\theta_{1}\left(\sigma_{i}\right)^{l} \theta_{2}}\right) .
$$

The skein relations and Lemma 4 are very useful for concrete computations of link polynomials, especially when $M \lesssim 3$.

Now we consider a particular class of words $\theta$ for which $L(\hat{\theta})$ can be worked out explicitly in a general fashion. With the help of (47) and Lemma 2, it is straightforward to prove the following:

Proposition 4. Assume $V(\Lambda) \otimes V(\Lambda)$ is multiplicity-free, and let

$$
\theta=\left(\sigma_{i_{1}}\right)^{l_{1}}\left(\sigma_{i_{2}}\right)^{l_{2}} \cdots\left(\sigma_{i_{n-1}}\right)^{l_{n-1}} \in B_{n}, \quad l_{1}, l_{2}, \ldots, l_{n-1} \in Z \text {, }
$$


with $\left(i_{i}, i_{2}, \ldots, i_{n-1}\right)$ an arbitrary permutation of $(1,2, \ldots, n-1)$. Then

$$
L(\widehat{\theta})=q^{-2 I_{2}(\Lambda)} \sum_{t=1}^{n-1} \prod_{t=1}^{l_{t}} \prod_{i=1}^{n-1}\left\{\sum_{\mu=1}^{M}\left[\varepsilon\left(\Lambda_{\mu}\right) q^{I_{2}\left(\Lambda_{\mu}\right) / 2}\right]^{l_{t}} D_{q}\left(\Lambda_{\mu}\right) / D_{q}(\Lambda)\right\} .
$$

It is worth noting that the tensor product $V(\Lambda) \otimes V(\Lambda)$ is always multiplicity-free when all the weights of $V(\Lambda)$ occur with unit multiplicity.

\section{Examples}

In this section we consider some concrete examples to illustrate the general theory developed in the last section. In particular, we study the rank two tensor representations of $U_{q}(g l(m))$, the spinor representation of $U_{q}(\operatorname{so}(2 m+1))$ and the minimal representation of $U_{q}\left(E_{8}\right)$, and determine corresponding link polynomials. For the sake of simplicity, we restrict ourselves to the special case discussed in Proposition 4.

1. Irreducible Rank Two Tensor Representations of $U_{q}(g l(m))$. The rank two tensor representations of $U_{q}(g l(m))$ are those obtained by deforming the rank two symmetric and antisymmetric tensor representations of $g l(m)$. They are furnished respectively by the irreducible $U_{q}(g l(m))$ modules $V\left(2 \varepsilon_{1}\right), V\left(\varepsilon_{1}+\varepsilon_{2}\right)$, where $\varepsilon_{i}, i=1,2, \ldots, m$ is the standard basis for the $g l(m)$ weight space with the invariant form

$$
\left(\varepsilon_{i}, \varepsilon_{j}\right)=\delta_{i j}, \quad \forall i, j .
$$

Let us consider $V\left(\varepsilon_{1}+\varepsilon_{2}\right)$ first. It can be shown that

$$
V\left(\varepsilon_{1}+\varepsilon_{2}\right) \otimes V\left(\varepsilon_{1}+\varepsilon_{2}\right)=\bigoplus_{\mu=0}^{2} V\left(\Lambda_{\mu}\right)
$$

with

$$
\Lambda_{0}=2\left(\varepsilon_{1}+\varepsilon_{2}\right), \quad \Lambda_{1}=2 \varepsilon_{1}+\varepsilon_{2}+\varepsilon_{3}, \quad \Lambda_{2}=\varepsilon_{1}+\varepsilon_{2}+\varepsilon_{3}+\varepsilon_{4},
$$

and

$$
\varepsilon\left(\Lambda_{0}\right)=\varepsilon\left(\Lambda_{2}\right)=-\varepsilon\left(\Lambda_{1}\right)=+1
$$

Using the formula

$$
\rho=\frac{1}{2} \sum_{i=1}^{m}(m-2 i+1) \varepsilon_{i}
$$

we can easily see that

$$
\begin{aligned}
I_{2}\left(\varepsilon_{1}+\varepsilon_{2}\right) & =2(m-1), & & I_{2}\left(\Lambda_{0}\right)=4 m \\
I_{2}\left(\Lambda_{1}\right) & =4(m-1), & & I_{2}\left(\Lambda_{2}\right)=4(m-3) .
\end{aligned}
$$

The $q$-dimensions of $V\left(\varepsilon_{1}+\varepsilon_{2}\right)$ and the $V\left(\Lambda_{\mu}\right)$ 's are given by

$$
D_{\dot{q}}\left(\varepsilon_{1}+\varepsilon_{2}\right)=\frac{\left(q^{m-1}-q^{1-m}\right)\left(q^{m}-q^{-m}\right)}{\left(q-q^{-1}\right)\left(q^{2}-q^{-2}\right)}
$$




$$
\begin{gathered}
D_{q}\left(2 \varepsilon_{1}+2 \varepsilon_{2}\right)=\frac{\left(q^{m-1}-q^{1-m}\right)\left(q^{m}-q^{-m}\right)^{2}\left(q^{m+1}-q^{-1-m}\right)}{\left(q-q^{-1}\right)\left(q^{2}-q^{-2}\right)^{2}\left(q^{3}-q^{-3}\right)} \\
D_{q}\left(2 \varepsilon_{1}+\varepsilon_{2}+\varepsilon_{3}\right)=\frac{\left(q^{m-2}-q^{2-m}\right)\left(q^{m-1}-q^{1-m}\right)\left(q^{m}-q^{-m}\right)\left(q^{m+1}-q^{-1-m}\right)}{\left(q-q^{-1}\right)^{2}\left(q^{2}-q^{-2}\right)\left(q^{4}-q^{-4}\right)} \\
D_{q}\left(\varepsilon_{1}+\varepsilon_{2}+\varepsilon_{3}+\varepsilon_{4}\right)=\frac{\left(q^{m-3}-q^{3-m}\right)\left(q^{m-2}-q^{2-m}\right)\left(q^{m-1}-q^{1-m}\right)\left(q^{m}-q^{-m}\right)}{\left(q-q^{-1}\right)\left(q^{2}-q^{-2}\right)\left(q^{3}-q^{-3}\right)\left(q^{4}-q^{-4}\right)}
\end{gathered}
$$

For any $\theta \in B_{n}$ of the special form (48), we can explicitly work out the corresponding link polynomial; it reads

$$
L(\hat{\theta})=q^{-2(m-1)}{ }_{t=1}^{n-1} l_{t=1}^{n-1} \prod_{q}\left(l_{t}\right)
$$

with

$$
\begin{aligned}
\Sigma_{q}\left(l_{t}\right)= & q^{2 l_{t}} \frac{\left(q^{m}-q^{-m}\right)\left(q^{m+1}-q^{-1-m}\right)}{\left(q^{2}-q^{-2}\right)\left(q^{3}-q^{-3}\right)} \\
& +(-)^{l_{t}} \frac{\left(q^{m-2}-q^{2-m}\right)\left(q^{m+1}-q^{-1-m}\right)}{\left(q-q^{-1}\right)\left(q^{4}-q^{-4}\right)} \\
& +q^{-4 l_{t}} \frac{\left(q^{m-3}-q^{3-m}\right)\left(q^{m-2}-q^{2-m}\right)}{\left(q^{3}-q^{-3}\right)\left(q^{4}-q^{-4}\right)}
\end{aligned}
$$

Now we study the module $V\left(2 \varepsilon_{1}\right)$. The tensor product $V\left(2 \varepsilon_{1}\right) \otimes V\left(2 \varepsilon_{1}\right)$ reduces to

with

$$
V\left(2 \varepsilon_{1}\right) \otimes\left(2 \varepsilon_{1}\right)=\bigoplus_{\mu=0}^{2} V\left(\Lambda_{\mu}\right)
$$

and

$$
\Lambda_{0}=4 \varepsilon_{1}, \quad \Lambda_{1}=3 \varepsilon_{1}+\varepsilon_{2}, \quad \Lambda_{2}=2 \varepsilon_{1}+2 \varepsilon_{2},
$$

$$
\varepsilon\left(\Lambda_{0}\right)=\varepsilon\left(\Lambda_{2}\right)=-\varepsilon\left(\Lambda_{1}\right)=+1
$$

The $I_{2}$ 's are given by

$$
\begin{array}{ll}
I_{2}\left(2 \varepsilon_{1}\right)=2(m+1), & I_{2}\left(\Lambda_{0}\right)=4(m+3), \\
I_{2}\left(\Lambda_{1}\right)=4(m+1), & I_{2}\left(\Lambda_{2}\right)=4 m
\end{array}
$$

and the $q$-dimensions read

$$
\begin{aligned}
D_{q}\left(2 \varepsilon_{1}\right) & =\frac{\left(q^{m+1}-q^{-m-1}\right)\left(q^{m}-q^{-m}\right)}{\left(q-q^{-1}\right)\left(q^{2}-q^{-2}\right)}, \\
D_{q}\left(4 \varepsilon_{1}\right) & =\frac{\left(q^{m+3}-q^{-m-3}\right)\left(q^{m+2}-q^{-m-2}\right)\left(q^{m+1}-q^{-m-1}\right)\left(q^{m}-q^{-m}\right)}{\left(q-q^{-1}\right)\left(q^{2}-q^{-2}\right)\left(q^{3}-q^{-3}\right)\left(q^{4}-q^{-4}\right)}, \\
D_{q}\left(3 \varepsilon_{1}+\varepsilon_{2}\right) & =\frac{\left(q^{m+2}-q^{-m-2}\right)\left(q^{m+1}-q^{-m-1}\right)\left(q^{m}-q^{-m}\right)\left(q^{m-1}-q^{1-m}\right)}{\left(q-q^{-1}\right)^{2}\left(q^{2}-q^{-2}\right)\left(q^{4}-q^{-4}\right)},
\end{aligned}
$$

and $D_{q}\left(2 \varepsilon_{1}+2 \varepsilon_{2}\right)$ is given in (53). Using the formulae (57)-(59) in Eq. (49) we again 
obtain an explicit expression for a polynomial for the link arising from a braid $\theta \in B_{n}$ defined by (48).

2. Spinor Representation of $U_{q}(\operatorname{so}(2 m+1))$. The spinor module of $U_{q}(\operatorname{so}(2 m+1))$ has the highest weight

$$
\Lambda=\frac{1}{2}\left(\varepsilon_{1}+\varepsilon_{2}+\cdots+\varepsilon_{m}\right) .
$$

Denoting this module by $V(\Lambda)$, we have

$$
V(\Lambda) \otimes V(\Lambda)=\bigoplus_{\mu=0}^{m} V\left(\Lambda_{m-\mu}\right)
$$

with

$$
\Lambda_{m-\mu}=\sum_{i=1}^{m-\mu} \varepsilon_{i}, \quad \varepsilon\left(\Lambda_{m-\mu}\right)=(-1)^{\mu(\mu+1) / 2} .
$$

The $q$-dimensions are given by

$$
\begin{aligned}
D_{q}(\Lambda)= & \prod_{i=1}^{m}\left(q^{m-i+1 / 2}+q^{i-m-1 / 2}\right) \\
D_{q}\left(\Lambda_{m-\mu}\right)= & \frac{q^{m+1 / 2}-q^{-m-1 / 2}}{q^{\mu+1 / 2}-q^{-1 / 2-\mu}} \\
& \cdot \prod_{i=1}^{m-\mu} \frac{\left(q^{m+1+\mu-i}-q^{i-m-1-\mu}\right)\left(q^{2 m+1-i}-q^{i-2 m-1}\right)\left(q^{2 m+2-i}-q^{i-2 m-2}\right)}{\left(q^{m+1-\mu-i}-q^{i-m-1+\mu}\right)\left(q^{2 m+2-2 i}-q^{2 i-2 m-2}\right)\left(q^{2 m+3-2 i}-q^{2 i-2 m-3}\right)} .
\end{aligned}
$$

The $I_{2}$ 's can also be worked out easily, and we get

$$
\begin{aligned}
I_{2}(\Lambda) & =m(2 m+1) / 4, \\
I_{2}\left(\Lambda_{m-\mu}\right) & =(m-\mu)(m+\mu+1) .
\end{aligned}
$$

Applying Eqs. (60)-(63) to (49) yields an explicit expression for $L(\hat{\theta})$, which will not be spelt out here.

3. The Minimal Representation of $U_{q}\left(E_{8}\right)$. Our final example is the exceptional quantum group $U_{q}\left(E_{8}\right)$. Recently the other exceptional quantum groups have been studied in their minimal representations [14]. Here we also consider only the minimal representation of $U_{q}\left(E_{8}\right)$.

Let us first introduce some notation. We choose the following simple root system for the Lie algebra $E_{8}$ :

$$
\begin{aligned}
& \frac{1}{2}\left[\varepsilon_{8}+\varepsilon_{1}-\left(\varepsilon_{2}+\varepsilon_{3}+\varepsilon_{4}+\varepsilon_{5}+\varepsilon_{6}+\varepsilon_{7}\right)\right], \varepsilon_{2}-\varepsilon_{1}, \varepsilon_{3}-\varepsilon_{2}, \varepsilon_{4}-\varepsilon_{3}, \\
& \varepsilon_{5}-\varepsilon_{4}, \varepsilon_{6}-\varepsilon_{5}, \varepsilon_{7}-\varepsilon_{6}, \varepsilon_{1}+\varepsilon_{2} .
\end{aligned}
$$

The corresponding set of positive roots is then given by

$$
\Phi^{+}=\left\{ \pm \varepsilon_{i}+\varepsilon_{j}, \frac{1}{2}\left(\varepsilon_{8}+\sum_{k=1}^{7}(-1)^{a(k)} \varepsilon_{k}\right) \mid 1 \leqq i<j \leqq 8, \sum_{k=1}^{7} a(k) \equiv 0(\bmod 2)\right\},
$$

from which we obtain, for the half-sum of the positive roots

$$
\rho=23 \varepsilon_{8}+6 \varepsilon_{7}+5 \varepsilon_{6}+4 \varepsilon_{5}+3 \varepsilon_{4}+2 \varepsilon_{3}+\varepsilon_{2} .
$$


The minimal irreducible representation of $U_{q}\left(E_{8}\right)$ is 248-dimensional, and is furnished by the irreducible model $V(\Lambda)$ with the highest weight

$$
\Lambda=\varepsilon_{8}+\varepsilon_{7} .
$$

The tensor product of $V(\Lambda)$ with itself reduces to $[11,15]$

$$
V(\Lambda) \otimes V(\Lambda)=\bigoplus_{\mu=0}^{4} V\left(\Lambda_{\mu}\right),
$$

with

$$
\Lambda_{0}=0, \quad \Lambda_{1}=\varepsilon_{8}+\varepsilon_{7}, \quad \Lambda_{2}=2 \varepsilon_{8}, \quad \Lambda_{3}=2\left(\varepsilon_{8}+\varepsilon_{7}\right), \quad \Lambda_{4}=2 \varepsilon_{8}+\varepsilon_{7}+\varepsilon_{6},
$$

and

$$
\varepsilon\left(\Lambda_{0}\right)=\varepsilon\left(\Lambda_{2}\right)=\varepsilon\left(\Lambda_{3}\right)=-\varepsilon\left(\Lambda_{1}\right)=-\varepsilon\left(\Lambda_{4}\right)=+1 .
$$

The dimensions of the $V\left(\Lambda_{\mu}\right)$ 's are respectively given by

$$
\begin{gathered}
\operatorname{dim} V\left(\Lambda_{0}\right)=1, \quad \operatorname{dim} V\left(\Lambda_{1}\right)=248, \\
\operatorname{dim} V\left(\Lambda_{2}\right)=3875, \quad \operatorname{dim} V\left(\Lambda_{3}\right)=27000, \\
\operatorname{dim} V\left(\Lambda_{4}\right)=30380 .
\end{gathered}
$$

It is fairly straightforward to work out the $I_{2}$ 's. They read

$$
\begin{aligned}
I_{2}(\Lambda) & =I_{2}\left(\Lambda_{1}\right)=60, & & I_{2}\left(\Lambda_{0}\right)=0, \\
I_{2}\left(\Lambda_{2}\right) & =96, \quad I_{2}\left(\Lambda_{3}\right)=124, & & I_{2}\left(\Lambda_{4}\right)=120 .
\end{aligned}
$$

Using Eqs. (67)-(70) and (72) in formula (49) we arrive at

$$
L(\hat{\theta})=q^{-60} \sum_{t=1}^{n-1} l_{t} \prod_{t=1}^{n-1} \Sigma_{q}\left(l_{t}\right)
$$

with

$$
\begin{aligned}
\Sigma_{q}\left(l_{t}\right)= & \left\{q^{-60 l_{t}}+\left(-q^{-30}\right)^{l_{t}} D_{q}(\Lambda)+q^{-12 l_{t}} D_{q}\left(\Lambda_{2}\right)\right. \\
& \left.+q^{2 l_{t}} D_{q}\left(\Lambda_{3}\right)+(-1)^{l_{t}} D_{q}\left(\Lambda_{4}\right)\right\}\left(D_{q}(\Lambda)\right)^{-1},
\end{aligned}
$$

where $\theta \in B_{n}$ is of the special form (48). For example, when the trefoil knot $\hat{\theta}_{t}$ is considered, we have

Thus

$$
\theta_{t}=\sigma_{1}^{3} \in B_{2}
$$

$$
\begin{aligned}
L\left(\hat{\theta}_{t}\right) & =q^{-180} \Sigma_{q}(3) \\
& =\left(D_{q}\left(\Lambda_{1}\right)\right)^{-1} q^{-180}\left\{q^{-180}-q^{-90} D_{q}\left(\Lambda_{1}\right)+q^{-36} D_{q}\left(\Lambda_{2}\right)+q^{6} D_{q}\left(\Lambda_{3}\right)-D_{q}\left(\Lambda_{4}\right)\right\} .
\end{aligned}
$$

The $q$-dimensions are given by

$$
D_{q}\left(\Lambda_{\mu}\right)=\prod_{\alpha \in \Phi^{+}} \frac{q^{\left(\Lambda_{\mu}+\rho, \alpha\right)}-q^{-\left(\Lambda_{\mu}+\rho, \alpha\right)}}{q^{(\rho, \alpha)}-q^{-(\rho, \alpha)}}, \quad \mu=1,2,3,4,
$$

with $\Phi^{+}$and $\rho$ given by (65) and (66) respectively. Although we could expand the products in (76) explicitly, the final expressions are messy and not very illuminating. 
We note that the link polynomial assoicated with $V(\Lambda)$ satisfies a fifth-order skein relation; of course the general result of Lemma 4 holds here also, that the polynomial of the connected sum of a finite number of links is the product of the corresponding link polynomials.

\section{Conclusions}

A systematic method has been developed for constructing quantum group invariants. As an application, we have used the method to construct a general formula for link polynomials. Our formula agrees with that of Reshetikhin obtained through a different approach [10], but is more explicit, and the theory of quantum group invariants we have developed makes the derivation very simple. When applied to particular representations of the quantum groups $U_{q}\left(E_{8}\right)$ etc., this formula has yielded corresponding link polynomials.

As is well known, the Gelfand invariants of ordinary Lie algebras play a crucial role in their representation theory. However, for quantum groups the analogous invariants have not previously been determined (except for the case of $U_{q}(\operatorname{sl}(2)$ ) [1]). The method developed in this paper allows us to construct such invariants in a systematic way; results will be reported in a separate publication [16].

Acknowledgement. Financial support from the Australian Research Council is acknowledged.

\section{References}

1. Jimbo, M.: Commun. Math. Phys. 102, 247 (1986); Lett. Math. Phys. 10, 63 (1985); 11, 247 (1986)

2. Drinfeld, V. G.: Proc. ICM Berkeley 1986. Vol. 1, pp. 798-820

3. Baxter, R. J.: Exactly solved models in statistical mechanics. New York: Academic Press 1982

4. Alvarez-Gaumé, L., Gomes, C., Sierra, G.: Phys. Lett. B220, 142 (1989); for a review of conformal field theories see Moore, G., Seiberg, N.: Commun. Math. Phys. 123, 177 (1989)

5. Turaev, V. G.: Invent. Math. 92,527 (1988)

6. Wadati, M., Deguchi, T., Akutsu, Y.: Phys. Rep. 180, 248 (1989)

7. Witten, E.: Commun. Math. Phys. 121, 351 (1989)

8. Witten, E.: Gauge theories and integrable lattice models. Princeton University preprint IASSNS-HEP-89/11

9. Jones V. F. R.: Baxterization. Australian National University Research Report CMA-R23-89

10. Reshetikhin, N. Yu.: Quantized universal enveloping algebras, the Yang-Baxter equation and invariants of links. I, II. L.O.M.I (Leningrad) preprints E-4-87, E-17-87

11. Rosso, M.: Commun. Math. Phys. 117, 581 (1988)

12. Kauffman, L. H.: On Knots, pp. 47-48. Princeton NJ: Princeton University Press 1987

13. Wadati, M., Yamada, Y., Deguchi, T.: J. Phys. Soc. Jpn 58, 1153 (1989)

14. Kuniba, A.: Quantum $R$-matrix for $G_{2}$ and a solvable 173 vertex model. Kyoto preprint, RIMS-664 (1989); Koh, I. G., Ma, Z. Q.: Exceptional quantum groups. ICTP preprint, IC/89/259

15. Slansky, R.: Phys. Rep. 79, 1 (1981)

16. Gould, M. D., Zhang, R. B., Bracken, A. J.: Generalized Gelfand invariants and characteristic identities for quantum groups, J. Math. Phys. (in press) 
